Effect of gel at i $n$ on the water di spersi on and cent $r$ i fugal pur i fi cat i on of singl e-wal I ed car bon nanot ubes

\begin{tabular}{|l|l|}
\hline 著者 & Kazi Hani um Nari a, M eno Tet su \\
\hline $\begin{array}{l}\text { j ournal or } \\
\text { publ i cat i on ti t l e }\end{array}$ & Japanese Journal of Appl i ed Physi cs \\
\hline vol une & 55 \\
\hline number & $1 S$ \\
\hline page r ange & 01AEO4 \\
\hline year & 2015- 11-05 \\
\hline 出版者 & Japan Soci et y of Appl i ed Physi cs \\
\hline 権利 & ( C) 2016 The Japan Soci et y of Appl i ed Physi cs \\
\hline URL & ht t p: //hdl . handl e. net /10297/9845 \\
\hline
\end{tabular}




\title{
Effect of gelatin on the water dispersion and centrifugal purification of single-walled carbon nanotubes
}

\author{
Kazi Hanium Maria and Tetsu Mieno* \\ Graduate School of Science and Technology, Shizuoka University, Shizuoka 422-8529, Japan \\ *E-mail: sptmien@ipc.shizuoka.ac.jp
}

\begin{abstract}
We report a convenient and effective procedure for the water dispersion and purification of single-walled carbon nanotubes (SWNTs). The purification procedure involves a combination of dispersion and centrifugation, in which gelatin; an environmentally friendly material is used as a dispersing agent. It has been found that an aqueous solution of gelatin effectively disperses SWNTs for more than a month. Another advantage of using gelatin as a dispersing agent is that it can be easily removed by washing with water and filtration. The centrifugation procedure employs a centrifugal force of about 2500 times the gravitational force to separate the particles. Although carbonaceous and metallic impurities usually have higher density than SWNTs in arc-produced carbon soot, the centrifugation can easily remove impurities leaving undamaged SWNTs in solution when appropriate centrifugal force and a centrifugation time are used. Centrifugation is carried out for three times to sufficiently remove impurities. Finally, the SWNTs are separated from the gelatin by heating in water and filtering.
\end{abstract}




\section{Introduction}

Single-walled carbon nanotubes (SWNTs), which have a nanometer-scale diameter and a high aspect ratio, exhibit excellent mechanical, electrical and magnetic properties, and are a promising material for a wide range of applications. ${ }^{1-3)}$ SWNTs usually form stable bundles owing to van der Waals interactions, making them difficult to disperse in many solvents. ${ }^{4)}$ This lack of solubility and the difficulty of manipulating them in solvents limit the use of SWNTs. Therefore, to apply SWNTs in a range of fields, it is necessary to develop dispersion techniques for SWNTs. The major successful approaches to disperse SWNTs to date have involved their chemical modification, which would considerably alter their desirable properties. ${ }^{5-7)}$ In comparison, polymer wrapping methods can be used to cover the nanotube surface and do not affect the physical properties of SWNTs while enabling their dispersion in aqueous solutions. ${ }^{8-12)}$ However these polymers and surfactants would remain in the sample and they would make poor purity in the samples. Therefore, easily decomposable polymer is a noteworthy factor for the dispersion of the SWNTs and also safe nanotube materials are desired related to the nano-bioscience and technology. In this work, we used gelatin, an environmentally friendly material to wrap the surface of SWNTs enabling their dispersion in water. ${ }^{13)}$

To obtain physical and chemical characteristic of SWNTs and to utilize them to wide applications, their purification is very important. As-prepared SWNTs are contaminated with metal catalysts (which are used in the growth process) and amorphous carbon (a by-product of 
SWNT growth). The presence of these impurities hinders their applications. Various purification methods for SWNTs have been extensively investigated. ${ }^{14-17)}$ Thermal treatment can easily remove the amorphous carbon. But the metal catalyst remains in the sample as their burning temperature is higher than the SWNTs. Acid oxidation treatments are the commonly used purification method which is effective in removing amorphous carbon and the metal catalysts. At the same time, this method damages the structure of SWNTs and resulting in poor mechanical properties and poor conductivity of SWNTs. Furthermore, large numbers of SWNTs are also etched away along with amorphous carbon, which leads to a low yield. Microwave heating treatments caused a loss of SWNTs and also led to additional wall damage. Therefore, nondestructive methods of separating impurities from SWNTs are of great importance.

With this background, we report a convenient and efficient purification method using a combination of dispersion by sonication and centrifugation. Though the centrifugal purification is required longer treatment compared to other methods, this process leads to removal of most of the impurities, and the purified SWNTs are found to be less bundalized and undamaged. Under a low centrifugation force and repeated centrifugation, highly dispersed SWNTs are precipitated as sediment while impurities remain in the solution. By decanting the supernatant, purified SWNTs can be collected. The main advantage of this method is that the impurities can be collected from the centrifuge tube after each step of the centrifugation process. 


\section{Experimental methods}

SWNTs were prepared in our laboratory by the bipolar pulsed arc discharge method. ${ }^{18)}$ SWNT sample (Nanostructure \& Amorphous Materials, diameter: 1-2 nm, length: 5-30 $\mu \mathrm{m}$, purity: 90\%) was also used as recieved to check the dispersion ability. Gelatin was purchased from Wako Pure Chemical Industries (product No. 077-03155, Wako 1st Grade, appearance: yellowish brown and crystalline powder) and used as received. The raw carbon soot (5 mg) and gelatin (20 mg) were mixed with $20 \mathrm{ml}$ pure water (Wako Pure Chemical Industries). Then the mixture was treated using an ultrasonic homogenizer equipped with a microtip sonotrode (Vibra Cell, Sonics \& Materials VC $130 f=20 \mathrm{kHz}, 6 \mathrm{~mm} \phi$ probe). The homogenizer was intermittently operated at an input power of $20 \mathrm{~W}$ for $60 \mathrm{~min}$ and the duty ratio was $60 \%$. A black gelatin-SWNT dispersion was obtained which remained well dispersed after more than a month at room temperature. A UV-visible spectrometer (JASCO V-630) was used to examine the dispersion stability of SWNTs-gelatin solution. For this experiment, $1 \mathrm{mg}$ of raw soot synthesized by the bipolar pulsed arc discharge and $5 \mathrm{mg}$ of gelatin were mixed with $10 \mathrm{ml}$ of pure water to make the sample. The sample was placed in a quartz cuvette $\left(1.0 \times 1.0 \times 4.5 \mathrm{~cm}^{3}\right)$ and time variation of absorbance (Abs) at a wavelength of $233 \mathrm{~nm}$ was measured for $4 \mathrm{~h}$. Here the wavelength was chosen corresponding to the maximum absorbance region of the UV-visible spectra.

Centrifugation of a dispersed solution of $1 \mathrm{mg}$ SWNT-containing soot and $2.5 \mathrm{mg}$ gelatin in $50 \mathrm{ml}$ pure water was performed at a low centrifugation force of $2500 \mathrm{G}$ for $2 \mathrm{~h}$. The centrifuge 
machine (Tomy Suprema 21) was equipped with an angle rotor with eight centrifuge tubes (NA-4HS, $50 \mathrm{ml} \times 8$ ). The sediment collected by decantation of the supernatant was redispersed in pure water by sonication $(30 \mathrm{~min})$ and centrifuged again. This dispersion-centrifugation-decantation process was performed three times. Finally, the supernatant and sediment were collected separately. To remove the gelatin, both were dispersed in pure water again, heated for $15 \mathrm{~min}$ at temperature of $80{ }^{\circ} \mathrm{C}$, filtered with a membrane filter paper with hole size of $0.025 \mu \mathrm{m}$ and washed with pure water. After the filtration, purified SWNTs are collected from the filter and most of the gelatin is drawn off with the filtrate.

A transmission electron microscope (TEM, JEOL JEM1400, accelerating voltage $100 \mathrm{kV}$ ) was used to observe the morphology of the SWNTs. Thermogravimetric (TG) and differential thermal analyses (DTA) of the SWNTs were carried out using a Rigaku Thermoplus Analyzer (TG8120), where about $2.5 \mathrm{mg}$ samples were heated at a heating rate of $10{ }^{\circ} \mathrm{C} / \mathrm{min}$ in air from room temperature to $800{ }^{\circ} \mathrm{C}$. Micro Raman spectra were obtained with a Raman spectrometer (JASCO NR-1800) using a laser for excitation $(\lambda=532 \mathrm{~nm})$.

\section{Results and discussion}

To observe the dispersion state of the wrapped SWNTs in water, one drop of the solution was placed on a copper grid coated with a collodion film for TEM observation. Figure 1 shows the TEM images of the raw soot and purchased SWNT sample and their wrapped conditions. It is seen that the gelatin uniformly wrapped around the surface of the nanotubes, by which the 
SWNTs are opaque and the surfaces are rough as compared with the unwrapped SWNTs. High magnification TEM image of the bipolar pulsed arc discharge soot shows that the unwrapped bundles of SWNTs consists of 1-12 SWNTs and the tube diameters are approximately $1.0-1.5$ nm. ${ }^{18)}$ But after the polymer wrapping process, the polymer covering outside the SWNTs bundles is clearly demonstrated even in the low magnification TEM image, and it is difficult to determine the nanotube diameter inside the polymer wrapping. Thus the thickness of the polymer wrapping may distinguish the wrapped bundle from the larger diameter bundle. The wrapping mechanism of polymer is believed to be driven largely by thermodynamics to eliminate hydrophobic interface between the nanotubes and the aqueous medium. In case of raw soot, it is also observed that SWNTs entangle with amorphous carbon and metal catalyst particles even after the gelatin-wrapping. Usually SWNT bundles emerge from the metallic nanoparticles during the production. Gelatin can also disperse the metal catalyst and amorphous carbon by wrapping. Additionally, it is worth noting that the dried gelatin-wrapped SWNTs are easily re-dissolved in water by sonication.

Figure 2 shows the time variation of absorption spectrum for gelatin-SWNT dispersing liquid at a wavelength of $233 \mathrm{~nm}$. It is noticed that the absorbance started to decrease from 4.27 to 3.48 abs within more than $1 \mathrm{~h}$ and then show a stable nature with the increase of time. The value of absorbance remains stable from 3.40 to 3.46 even after the one month of dispersion which indicates the stability of gelatin-SWNT dispersion. 
The SWNTs have a tendency to aggregate owing to the attractive interaction between their sidewalls. The resulting hydrophobic nature is responsible for their poor solubility in water, and SWNTs are precipitated in water as shown in Figs. 3(a) and 3(b). However, SWNTs can be hydrated by gelatin, which can wrap around their sidewalls as schematically shown in Fig. 4 . Most polymer-wrapping methods depend on the physical adsorption of the polymer on the surface of the SWNTs to increase the solubility of the nanotubes. Gelatin is a natural water-soluble biopolymer that is composed of 18 types of amino acid chains. ${ }^{19,20)}$ The amino acid chains of the gelatin wrap around the sidewalls of the SWNTs through a hydrophobic-hydrophobic interaction. ${ }^{21,22)}$ It is conjectured that the interaction between the hydrophobic amino acids of the zwitterionic gelatin structure and the hydrophobic wall of the SWNTs modifies the SWNTs so that they include a hydrophilic group, allowing them to disperse in water as shown in Figs. 3(c) and 3(d). It was found that the dispersion remains stable for more than a month.

The stable gelatin-SWNT dispersion was centrifuged to remove the impurities. During the centrifugation, centrifugal force causes suspended particles of the solution move towards the bottom of the centrifuge tube depending on their specific gravity, size, and density. The effect of centrifugal force is higher on the dense particles and causes fast sedimentation. From our previous study, ${ }^{18)}$ the number of carbon particles $\left(N_{\mathrm{cp}}\right)$ and the number of SWNT bundles $\left(N_{\mathrm{ntb}}\right)$ are counted from the TEM images with the magnification $(\times 50000)$ over an equal area of $1.44 \times$ 
$10^{6} \mathrm{~nm}^{2}$, and their ratio is calculated. Generally shapes of the carbon particles are spheroid where both metal catalyst particles and simple amorphous-like carbon particles are considered. It is found that the number ratio of SWNT bundles to carbon particles is about 0.55 to 0.60 . It can be assumed from this ratio that the density of carbon particles in the soot is higher than those of the SWNTs, and the dense carbon particles are dropped faster than the light SWNTs in the centrifugation. Moreover, from the TG-DTA analysis, it is observed that the raw soot contains more metal particles $(40 \mathrm{wt} \%)$ than the SWNTs $(34 \mathrm{wt} \%)$. Therefore, the catalyst particles reach sedimentation quickly as sedimentation speed is based on specific gravity.

Figure 5 shows a schematic of the dispersion-centrifugation-decantation procedure at different conditions. After the first centrifugation, the supernatant was collected by decantation where the supernatant was considered to be the upper $80 \%$ of the solution. The residual solution was deposited at the bottom of the centrifuge tube. The collected supernatant was redispersed with pure water for 15 min and again centrifuged and this process was performed several times to separate the impurity from SWNTs. There are a number of factors which affect the separating ability of a centrifuge such as polymer and nanotube concentration, rotation speed or force and centrifugation times. To optimize the better separation, we investigated centrifugation under a range of different centrifugal force and centrifugal times and it was found that the separation was dependent on the centrifugal force as well as the rotation time. In case of $1 \mathrm{~h}$ centrifugation at $2500 \mathrm{G}$, the centrifugation-decantation cycle was repeated for 5 times to get pure SWNTs. It was 
time consuming and several cycles might causes loss of the sample during the process. As the time increased to $2 \mathrm{~h}$, the sediment containing more pure SWNTs was found after 3 times centrifugation-decantation cycle. However, $3 \mathrm{~h}$ centrifugation led to precipitation almost all the SWNTs and metal catalyst, whereas the supernatant contains little amount of carbon nanoparticle. Centrifugation was also carried out with increasing force for $2 \mathrm{~h}$. Sediment separated after first centrifugation at $3500 \mathrm{G}$ for $2 \mathrm{~h}$ contains considerable amount of SWNTs along with the metal catalyst and few amount of SWNTs appeared in the supernatant. In case of centrifugation at $5000 \mathrm{G}$ for $2 \mathrm{~h}$, a large amount of SWNTs and metal catalyst dropped to the sediment and supernatant became transparent like water. From the above experiment, it is therefore confirmed that centrifugation at $2500 \mathrm{G}$ for $2 \mathrm{~h}$ made the better separation of SWNTs from the impurities as illustrated in Fig. 5. It is found that after the second time centrifugation at $2500 \mathrm{G}$ for $2 \mathrm{~h}$, almost all the metal particles were removed from the soot as sediment, whereas supernatant contains many SWNTs with amorphous carbon and few metal particles. Therefore, supernatant was third time centrifuged at $2500 \mathrm{G}$ for $2 \mathrm{~h}$ to separate the amorphous carbon. After the third time centrifugation, almost all the dispersed SWNTs had sunk to the bottom of the centrifuge tube, and the amorphous and short length nanotubes remained in the supernatant. It is conjectured that the gelatin concentration might affect the sedimentation of SWNTs. Before the second and third centrifugation, pure water is added with the supernatant. In this process, gelatin concentration is decreased, which decreases the dispersing ability of SWNTs resulting in coagulation of SWNTs. 
These bundled nanotubes could increase the sedimentation speed due to their higher specific gravity. As the mechanism of the sedimentation of SWNTs is not made clear, we will do more experiment selecting many centrifuge conditions and try to make clear the mechanism.

The final product was again dispersed in pure water, heated to $80{ }^{\circ} \mathrm{C}$ for about 5 min and filtered to remove the gelatin. It is known that the zwitterionic interactions of gelatin are $\mathrm{pH}$ and temperature dependent. The $\mathrm{pH}$ of the solution is decreased by adding pure water. Decrease in the $\mathrm{pH}$ and increase in temperature lead to the dissociation of the SWNTs from the gelatin in water. $^{23,24)}$

TEM images of the raw soot and the product after each step of the purification process are shown in Fig. 6. Figure 6(a) shows that raw soot is heavily contaminated with amorphous carbon and the metal catalyst in the SWNTs. The TEM image of the sediment collected from the first step of centrifugation [Fig. 6(b)] shows that it contains the maximum amount of the metal catalyst. In this step, SWNTs are separated from the heavy metal catalyst by remaining in the supernatant solution. Subsequently, three times centrifugation of this supernatant led to further separation of impurities. From Fig. 6(c), it is clear that the impurity has been removed from the nanotubes. However gelatin is present in the soot. Finally, gelatin was almost removed from SWNTs as shown in Fig. 6(d), which was obtained by filtration, heating and washing with water.

To verify the effectiveness of the purification process, TG-DTA measurement was carried out. The raw soot exhibits two gravimetric decreases at approximately 335 and $380{ }^{\circ} \mathrm{C}$ as shown 
in Fig. 7(a), which corresponds to the abundances of amorphous carbon and SWNTs, respectively. ${ }^{25)}$ The content of the remaining materials above $700{ }^{\circ} \mathrm{C}$ is more than $40 \mathrm{wt} \%$, which indicates the presence of the metal catalyst. TG curve of purified SWNTs soot shows the gradual transition below $300{ }^{\circ} \mathrm{C}$. This transition occurs due to the fact that the purified soot contains a fraction of nanotubes with polymer group and a little amorphous carbon which is oxidized at lower temperature. But the raw or as-produced unpurified nanotubes contain more metal catalyst, which makes the TG curve stable at lower temperature. A small weight loss about $24 \mathrm{wt} \%$ at approximately $340{ }^{\circ} \mathrm{C}$ is observed from the TG result of pure SWNTs soot as shown in Fig. 7(b). It is conjectured that this weight loss originates from the combustion of gelatin or carbonaceous impurities. The weight loss at approximately $400{ }^{\circ} \mathrm{C}$ in Fig. 7(b) might be due to the combustion of the SWNTs. Because purified soot becomes more compact after drying and contain small amount of metal catalyst and higher-purity SWNTs, which have higher combustion temperature owing to the absence of the metal catalyst. It was also observed that a thermally degraded carbon content of $17 \mathrm{wt} \%$ remained above a temperature of $800{ }^{\circ} \mathrm{C}$.

In the Raman spectra of SWNTs, the D-band (vibrational mode, $1345 \mathrm{~cm}^{-1}$ ), the tangential G-band $\left(1590 \mathrm{~cm}^{-1}\right)$, and the low-frequency radial breathing mode (RBM) in the range of 100-300 $\mathrm{cm}^{-1}$ are prominent features. ${ }^{26-28)}$ The Raman measurement was conducted for both the raw soot and the purified soot. As shown in Fig. 8, RBM peaks were observed at 170 and 187 $\mathrm{cm}^{-1}$ for both the raw soot and the purified soot, from which the SWNT diameters were 
calculated to be 1.46 and $1.32 \mathrm{~nm}$, respectively. The similar diameter distributions indicate that the structure of the SWNTs was not so damaged by the purification procedure. ${ }^{13)}$ Both samples have almost the same sharp peaks at $1590 \mathrm{~cm}^{-1}$, as shown in Fig. 8, which were assigned to the ordered phase in the graphite material. The peak intensity of the D-band, which was assigned to the disordered phase, was observed to be lower for the purified soot. The small $I_{\mathrm{D}} / I_{\mathrm{G}}$ ratio of the purified soot compared with that of the raw soot indicates the effective removal of impurities by centrifugation. The full width at half maximum (FWHM) values of the RBM and G-band peaks also provide information about damage to the sidewalls of SWNTs. ${ }^{29,30)}$ From our experimental results, it was observed that the peaks of both these bands have almost the same FWHM values, indicating that the centrifugation did not result in the significant formation of wall defects.

The purity of the purified SWNTs was evaluated from the TG-DTA experiment as analytically pure SWNT sample is not available. The metal content in the residue material decreased from $40 \mathrm{wt} \%$ in the starting material to $17 \mathrm{wt} \%$ in the purified material. The amount of SWNT content in the raw soot was $38 \mathrm{wt} \%$ which was increased to $62 \mathrm{wt} \%$ after the purification. In this study, $4.3 \mathrm{mg}$ of purified soot was obtained from $8 \mathrm{mg}$ of raw soot. Therefore, purification efficiency can be estimated to be $58 \%$. From the viewpoint of purification, the efficiency of purification is not so high but the obtained SWNTs are almost pure and undamaged.

\section{Conclusions}

We have developed a nondestructive centrifugation-based process for separating impurities from 
SWNTs using gelatin, an environmentally friendly material. Gelatin was used as a dispersing agent, because the interaction between a biopolymer and SWNTs in water leads to the effective separation of aggregated SWNTs, and because of the simple removal of the gelatin from the SWNTs by heating. The combination of gelatin dispersion and centrifugation is a straightforward method of obtaining highly pure and undamaged SWNTs. The another advantage of this process is that the metal catalyst can be collected from the residual soot. The degree of purity can be controlled by adjusting the centrifugation speed.

\section{Acknowledgements}

This study was supported by the Promotion of Nano-Biotechnology Research to as Support Aging Project from the Ministry of Education, Culture, Sports, Science and Technology, Japan. The TEM measurement and TG/DTA analysis were carried out at the Research Institute of Green Science and Technology, Shizuoka University. 


\section{References}

1) J. P. Metters and C. E. Banks, Vacuum 86, 507 (2012).

2) R. Saito, G. Dresselhaus, and M. S. Dresselhaus, Physical Properties of Carbon Nanotubes (Imperial College Press, London, 1998) Chap. 4, p. 59.

3) S. Bellucci, M. Cini, P. Onorato, and E. Perfetto, J. Phys: Condens. Matter 18, S2115 (2006).

4) B. Fei, H. Lu, Z. Hu, and J. H. Xin, Nanotechnology 17, 1589 (2006).

5) J. L. Zimmerman, R. K. Bradley, C. B. Huffman, R. H. Hauge, and J. L. Margrave, Chem. Mater. 12, 1361 (2000).

6) I. W. Chiang, B. E. Brinson, A. Y. Huang, P. A. Willis, M. J. Brownikowski, J. L. Margrave, R. E. Smalley, and R. H. Hauge, J. Phys. Chem. B 105, 8297 (2001).

7) V. Georgakilas, D. Voulgaris, E. Vazquez, M. Prato, D. M. Guldi, and A. Kukovecz, J. Am. Chem. Soc. 124, 14318 (2002).

8) M. J. O’Connell, P. Boul, L. M. Ericson, C. Huffman, Y. Wang, E. Haroz, C. Kuper, J. Tour, K. D. Ausman, and R. E. Smalley, Chem. Phys. Lett. 342, 265 (2001).

9) A. Star, D. W. Steuerman, J. R. Heath, and J. F. Stoddart, Angew. Chem., Int. Ed. 41, 2508 (2002).

10) Y. Kang and T. A. Taton, J. Am. Chem. Soc. 125, 5650 (2003).

11) B. Mc Carthy, J. N. Coleman, R. Czerw, A. B. Dalton, D. L. Carroll, W. J. Blau, Synth. Met. 
121, $1225(2001)$.

12) D. E. Hill, Y. Lin, A. M. Rao, L. F. Allard, and Y. P. Sun, Macromolecules 35, 9466 (2002).

13) T. Takahashi, K. Tsunoda, H. Yajima, and T. Ishii, Jpn. J. Appl. Phys. 43, 1227 (2004).

14) K. B. Shelimov, R. O. Esenaliev, A. G. Rinzler, C. B. Huffman, and R. E. Smalley, Chem. Phys. Lett. 282, 429 (1998).

15) B. Zhao, H. Hu, S. Niyogi, M. E. Itkis, M. A. Hamon, P. Bhowmik, S. M. Meier, and C. R. Haddon, J. Am. Chem. Soc. 123, 11637 (2001).

16) M. T. Martinez, M. A. Callejas, A. M. Benito, W. K. Maser, M. Cochet, J. M. Andres, J. Schreiber, O. Chauvet, and J. L. G. Fierro, Chem. Commun. 1000 (2002).

17) A. R. Harutyunyan, B. K. Pradhan, J. Chang, G. Chen, and P. C. Eklund, J. Phys. Chem. B 106, $8671(2002)$.

18) K. H. Maria and T. Mieno, Vacuum 113, 11 (2015).

19) P. V. Kozlov and G. I. Burdygina, Polymer 24, 651 (1983).

20) P. I. Rose, in Encyclopedia of Polymer Science and Engineering, 2nd ed. H. F. Mark, N. M. Bikales, C. G. Overberger, and G. Manges (Wiley, New York, 1987) Vol. 7, p. 488.

21) W. Zheng and Y. F. Zheng, Electrochem. Commun. 9, 1619 (2007).

22) S. Banerjee, M. G. Khan, and S. S. Wong, Chem. Eur. J. 9, 1898 (2003).

23) M. Djabourov and P. Papon, Polymer 24, 537 (1983).

24) I. Dranca and S. Vyazovkin, Polymer 50, 4859 (2009). 
25) S. Arepalli, P. Nikolaev, O. Gorelik, V. G. Hadjiev, W. Holmes, B. Files, and L. Yowell, Carbon 42, 1783 (2004).

26) A. Jorio, M. A. Pimenta, A. Souza-Filho, R. Saito, G. Dresselhaus, and M. S. Dresselhaus, New J. Phys. 5, 139 (2003).

27) J. Maultzch, H. Telg, S. Reich, and C. Thomsen, Phys. Rev. B 72, 205438 (2005).

28) H. Kataura, Y. Kumazawa, Y. Maniwa, I. Umezu, S. Suzuki, Y. Ohtsuka, and Y. Achiba, Synth. Met. 103, 2555 (1999).

29) D. Nishide, H. Kataura, S. Suzuki, K. Tsukagoshi, Y. Aoyagi, and Y. Achiba, Chem. Phys. Lett. 372, 45 (2003).

30) M. Monthioux, B. W. Smith, B. Burteaux, A. Claye, J. E. Fischer, and D. E. Luzzi, Carbon 39, 1251 (2001). 


\section{Figure captions:}

Fig.1. TEM images of (a) raw soot synthesized by the bipolar pulsed arc discharge, (b) gelatin-wrapped SWNT bundles synthesized by bipolar pulsed arc discharge (c) SWNTs sample (Nanostructure \& Amorphous Materials) and (d) gelatin-wrapped SWNT bundles (Nanostructure \& Amorphous Materials).

Fig.2. Absorbance versus time at $\lambda=233 \mathrm{~nm}$ to measure the stability of the gelatin-SWNT dispersion. Inset shows the UV absorption spectrum of the solution.

Fig.3. Photographs of (a) SWNT dispersion in water immediately after sonication, (b) SWNT precipitate in water after a few hours, (c) SWNT dispersion in gelatin-water solution immediately after sonication, and (d) a stable SWNT-gelatin dispersion after one month.

Fig.4. (Color online) Schematic showing wrapping of SWNTs with gelatin.

Fig.5. Schematic diagram of the dispersion-centrifugation-decantation procedure at different conditions.

Fig.6. TEM images of (a) raw soot, (b) sediment after the first centrifugation, (c) sediment after the final centrifugation, and (d) sediment from the (c) after the removal of gelatin by heating and filtration.

Fig.7. (Color online) TG-DTA results for (a) raw soot and (b) purified soot.

Fig.8. (Color online) Raman spectra of (a) raw soot and (b) purified soot after the removal of gelatin. The RBM modes are shown on the left side where corresponding diameters are written. 
Intensities of D-band $\left(I_{\mathrm{D}}\right)$ and G-band $\left(I_{\mathrm{G}}\right)$ are shown on the right side.

(a)

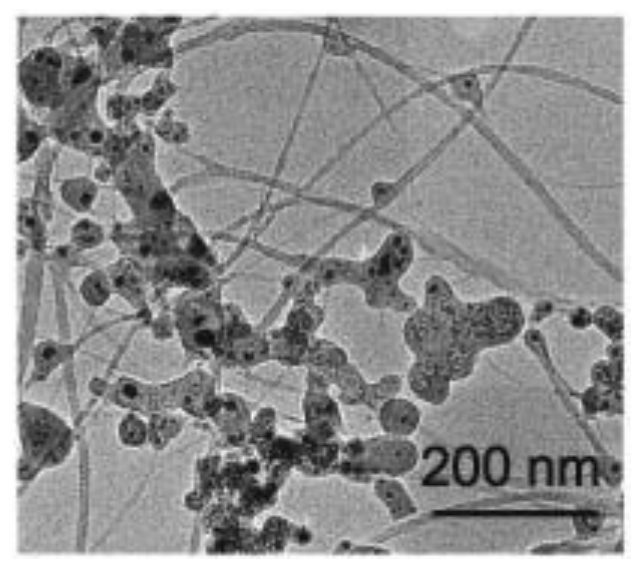

(c)

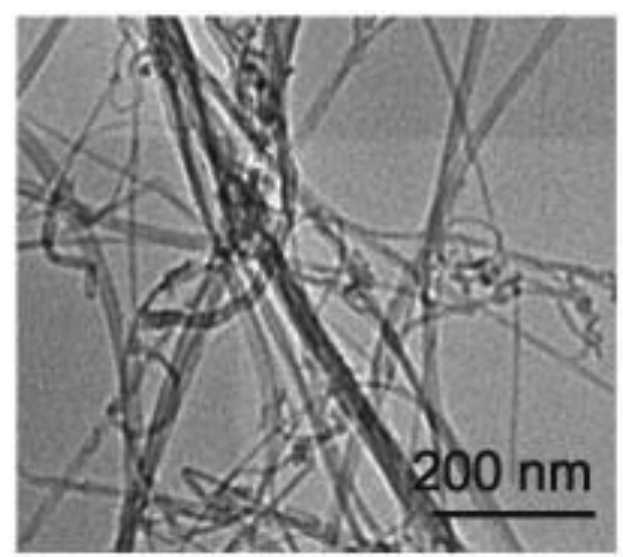

(b)

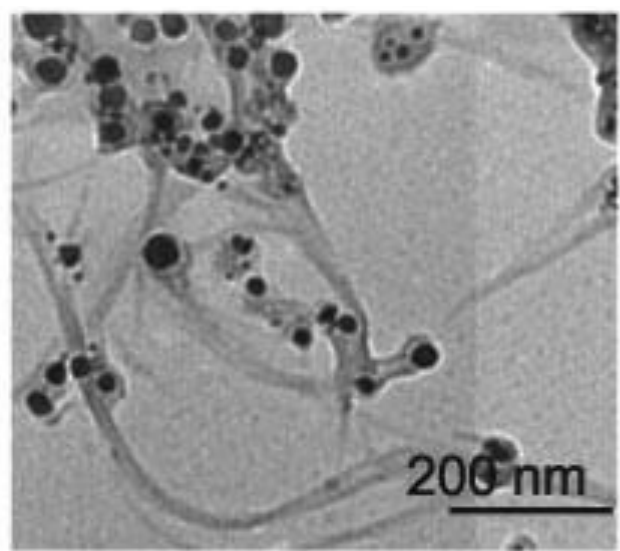

(d)

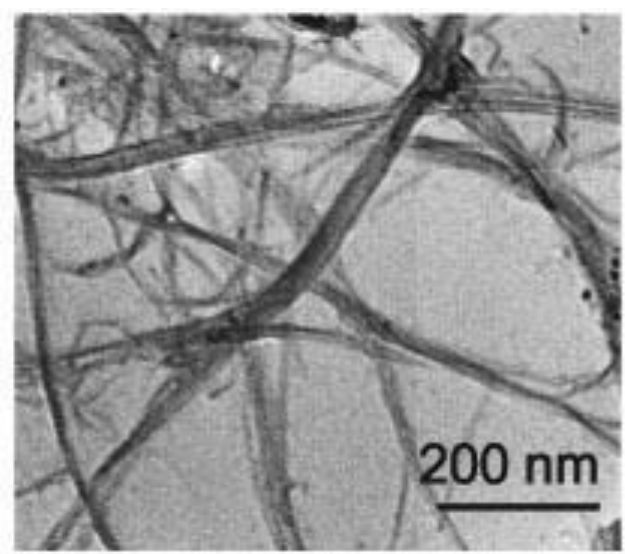

Fig.1. TEM images of (a) raw soot synthesized by the bipolar pulsed arc discharge, (b) gelatin-wrapped SWNT bundles synthesized by bipolar pulsed arc discharge (c) SWNTs sample (Nanostructure \& Amorphous Materials) and (d) gelatin-wrapped SWNT bundles (Nanostructure \& Amorphous Materials). 


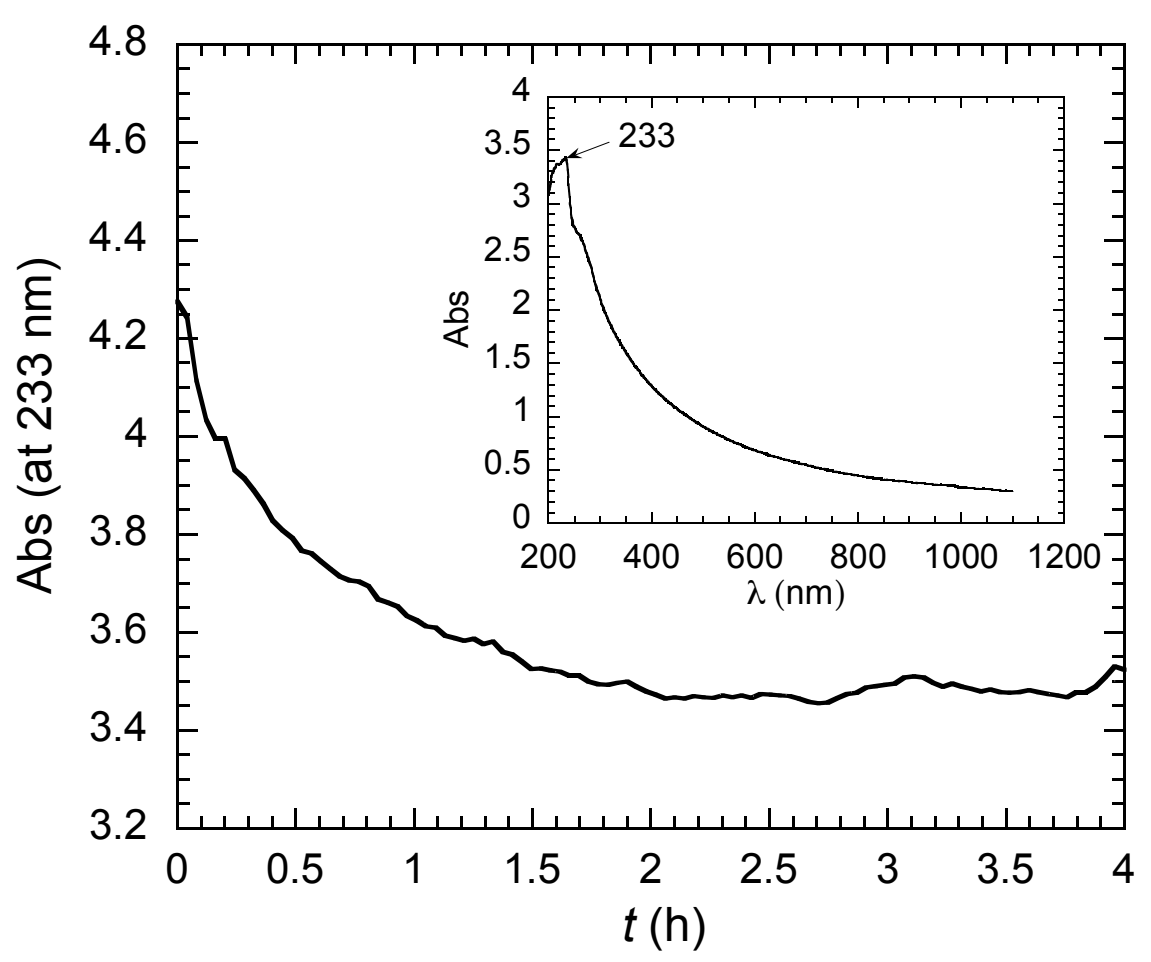

Fig.2. Absorbance versus time at $\lambda=233 \mathrm{~nm}$ to measure the stability of the gelatin-SWNT dispersion. Inset shows the UV absorption spectrum of the solution. 

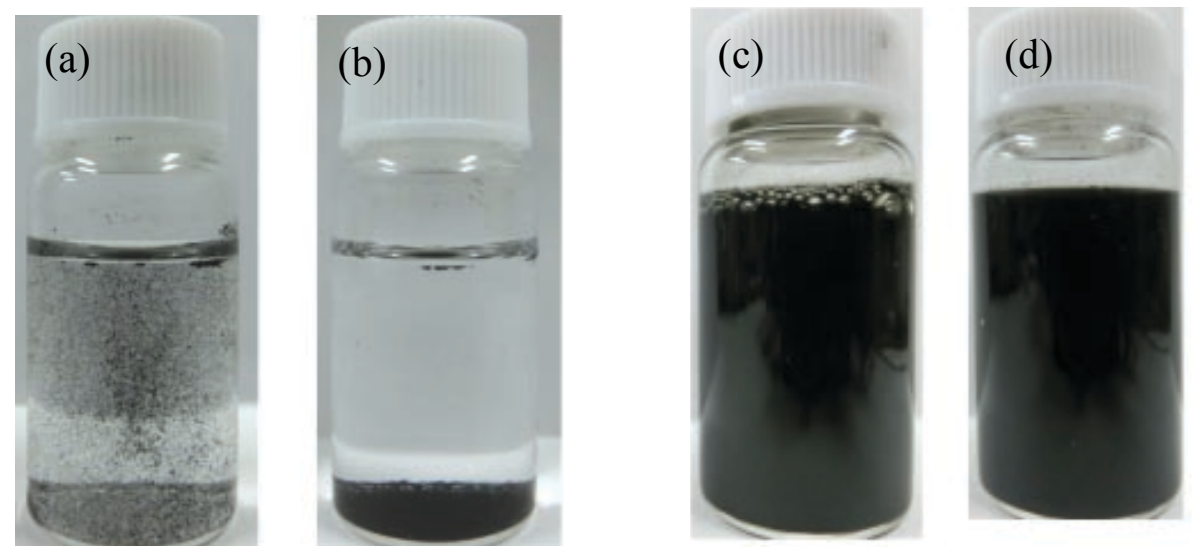

Fig.3. Photographs of (a) SWNT dispersion in water immediately after sonication, (b) SWNT precipitate in water after a few hours, (c) SWNT dispersion in gelatin-water solution immediately after sonication, (d) stable SWNT-gelatin dispersion after one month. 


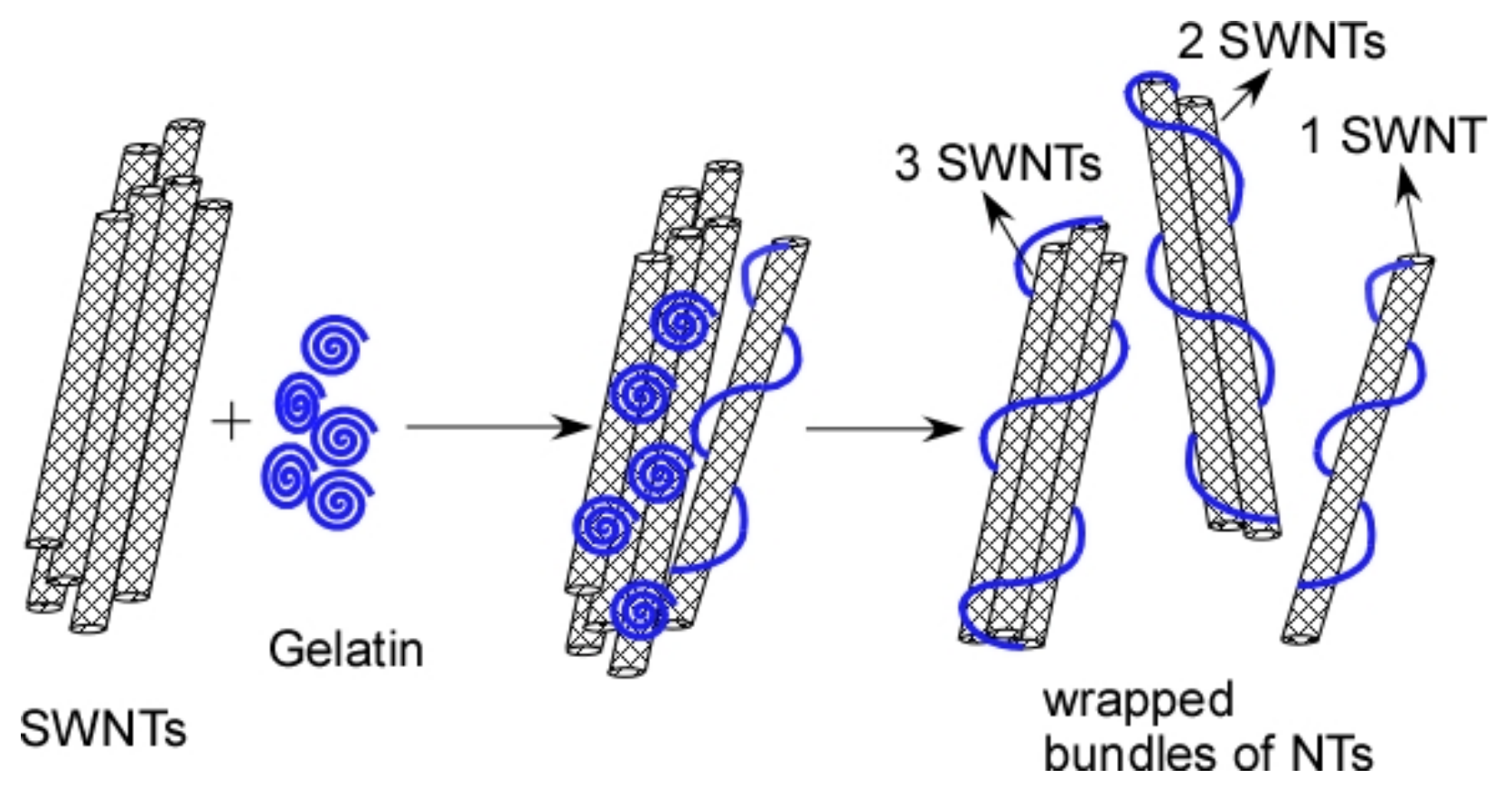

Fig.4. (Color online) Schematic showing wrapping of SWNTs with gelatin. 


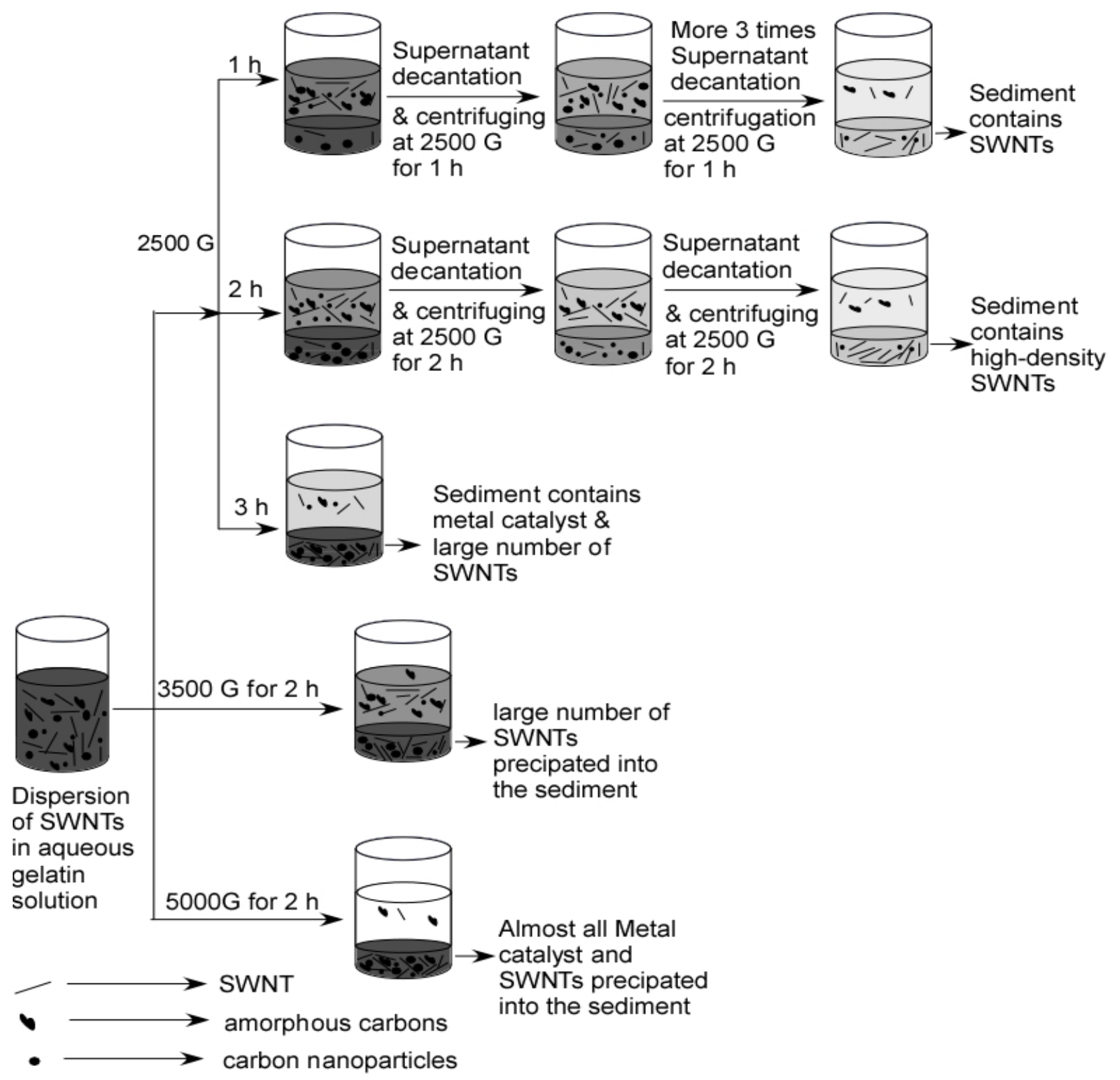

Fig.5. Schematic diagram of the dispersion-centrifugation-decantation procedure at different conditions. 
(a)

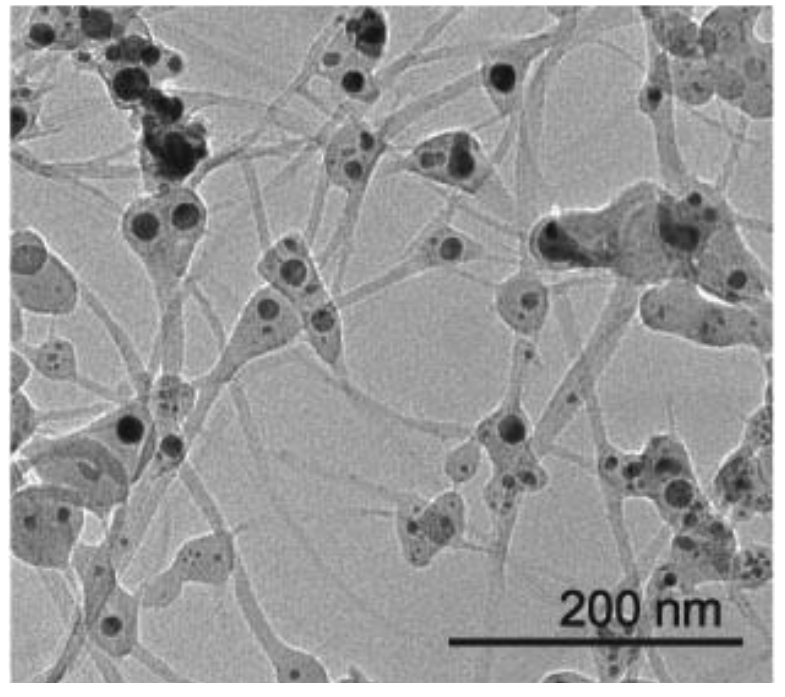

(c)

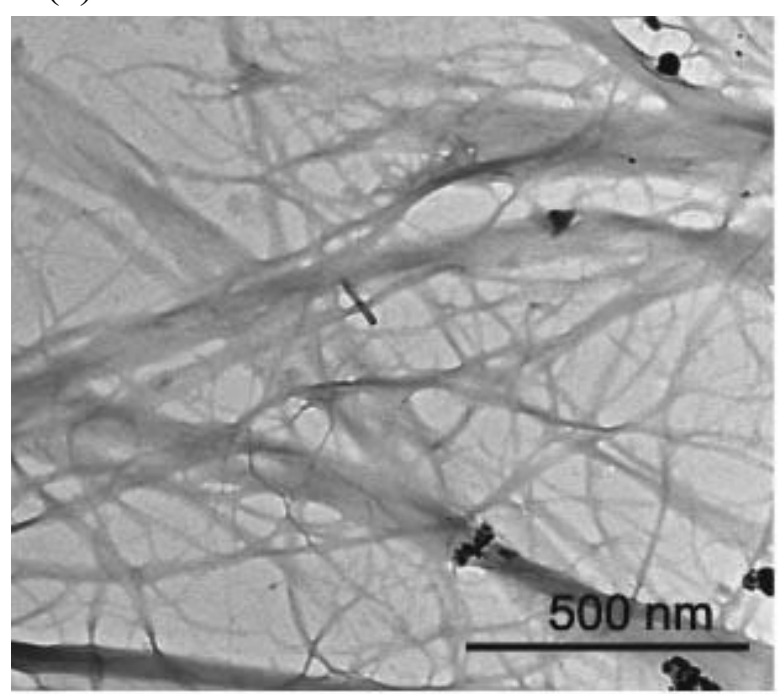

(b)

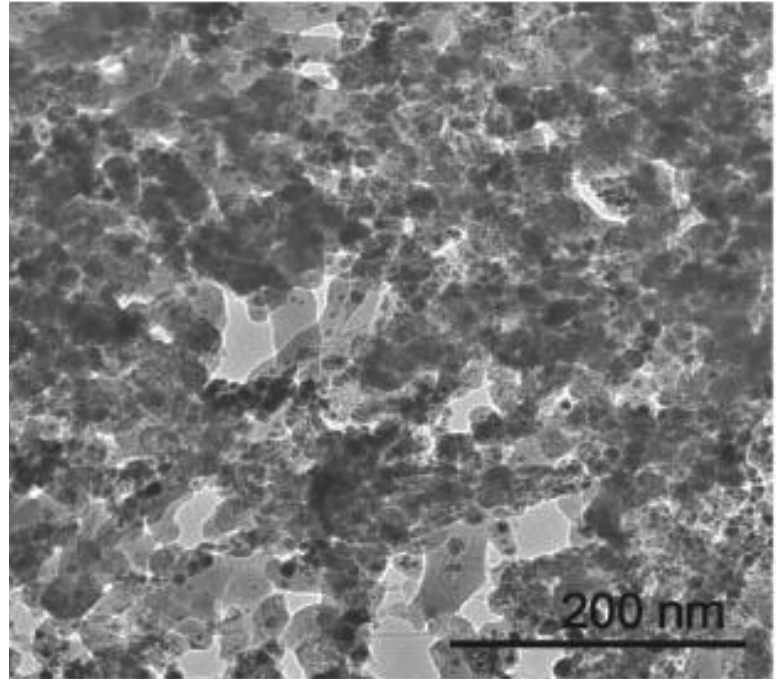

(d)

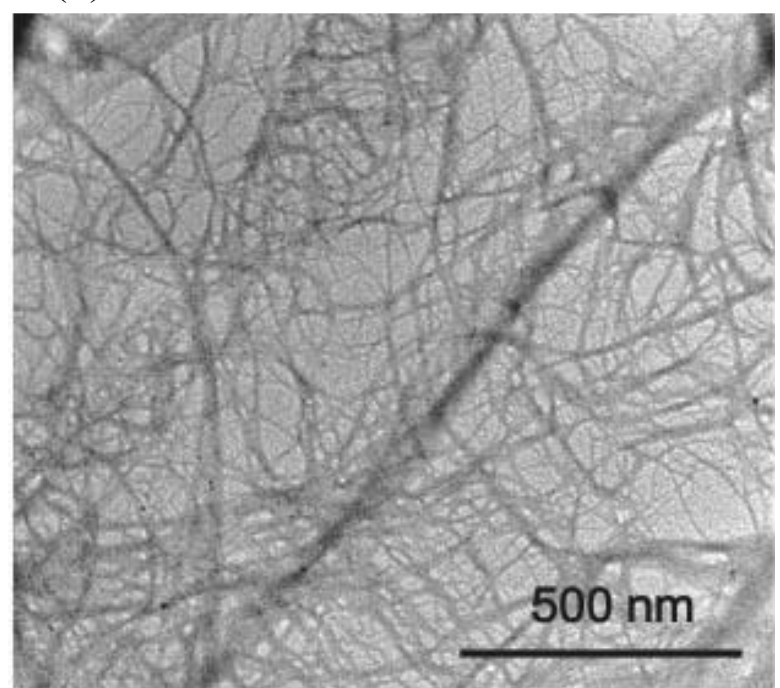

Fig.6. TEM images of (a) raw soot, (b) sediment after the first centrifugation, (c) sediment after the final centrifugation, and (d) sediment from the (c) after the removal of gelatin by heating and filtration. 

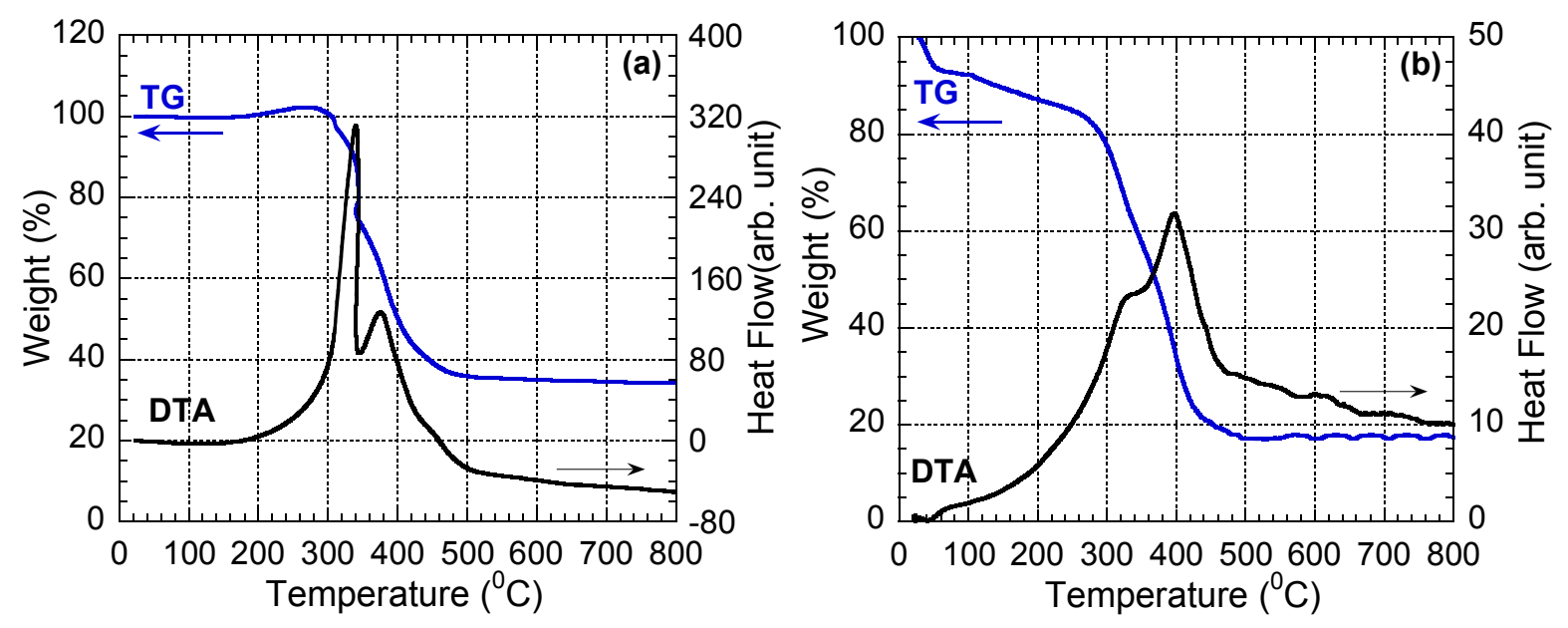

Fig.7. (Color online) TG-DTA results for (a) raw soot and (b) purified soot. 
(a) Raw soot
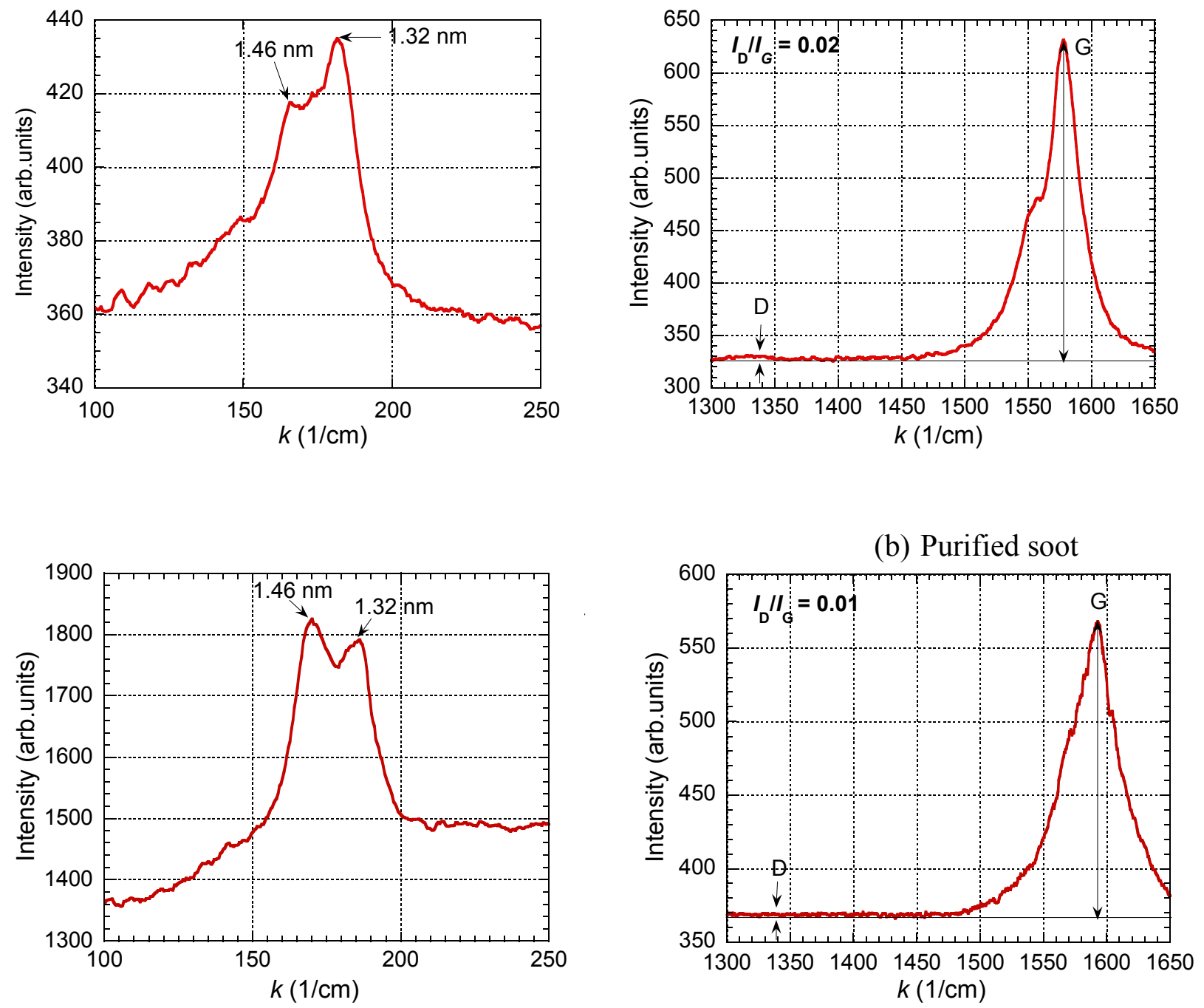

Fig.8. (Color online) Raman spectra of (a) raw soot and (b) purified soot after the removal of gelatin. The RBM modes are shown on the left side where corresponding diameters are written. Intensities of D-band $\left(I_{\mathrm{D}}\right)$ and G-band $\left(I_{\mathrm{G}}\right)$ are shown on the right side. 